WORKING PAPER $\cdot$ NO. 2020-56

Belief in Science Influences Physical

Distancing in Response to COVID-19 Lockdown Policies

Adam Brzezinski, Valentin Kecht, David Van Dijcke, and Austin L. Wright APRIL 2020

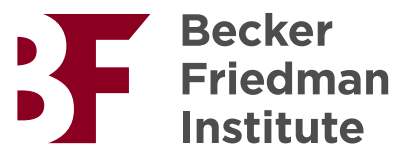




\title{
Belief in Science Influences Physical Distancing in Response to COVID-19 Lockdown Policies*
}

\author{
Adam Brzezinski ${ }^{1}$, Valentin Kecht $^{2}$, David Van Dijcke ${ }^{1,3}$, Austin L. Wright ${ }^{4}$ \\ 1 Department of Economics, University of Oxford \\ 2 Ettore Bocconi Department of Economics, Bocconi University \\ 3 Institute for New Economic Thinking at the Oxford Martin School, University of Oxford \\ 4 Harris School of Public Policy, University of Chicago
}

\begin{abstract}
Physical distancing reduces transmission risks and slows the spread of COVID-19. Local and regional governments in the United States have issued shelter-in-place policies to mandate physical distancing. Yet compliance with these policies is uneven and may be influenced by beliefs about science and topics of scientific consensus. We theorize that individuals skeptical about the human causes of climate change are less likely to comply with physical distancing orders. Using county-day measures of physical distancing derived from cellphone location data, we demonstrate that the proportion of people who stay at home after lockdown policies go into effect is significantly lower in counties with a high concentration of climate change skeptics. These results are consistent when we study how belief in science influences physical distancing across as well as within Democratic and Republican counties. Our findings suggest public health interventions and messaging about risks associated with COVID-19 that take into account local attitudes towards science may be more effective.
\end{abstract}

Keywords: COVID-19, physical distancing, belief in science, political partisanship.

Classification: Social Sciences/Economic Sciences (Major), Physical Sciences/Statistics (Minor).

JEL-Classification: I12, I18, H12, H75, D04.

${ }^{*}$ Corresponding author: David Van Dijcke (david.vandijcke@economics.ox.ac.uk). We thank SafeGraph for providing their data and support. We also thank Rick Van der Ploeg, Guido Deiana, and Cyrus Samii for support and discussions. 
Significance Statement Physical distancing reduces interpersonal transmission risks. Government policies that mandate physical distancing slow the spread of COVID-19. Local non-compliance with these shelter-in-place orders creates public health risks and may cause regional spread. We study how beliefs about science influence compliance with these local mandates in the United States through the use of location trace data. Our findings suggest climate change skepticism is associated with lower compliance with government mandates even within political parties. Policy interventions and public messaging about COVID-19 risks need to take into account scientific skepticism to increase effectiveness. 
Introduction Physical distancing reduces interpersonal transmission risks (Anderson et al., 2020 Bai et al., 2020: Viner et al., 2020). Government policies that mandate physical distancing slow the spread of COVID-19 (Hsiang et al., 2020). Local non-compliance with these shelterin-place orders creates public health risks and may cause regional spread (Chen et al., 2020; Lewnard and Lo, 2020). Understanding which local-level factors impact compliance is a first order public policy concern (Briscese et al., 2020) and informs evidence-based policy interventions to flatten the infection curve.

Recent research highlights several factors that influence compliance: partisanship (Allcott et al., 2020; Gadarian et al., 2020; Grossman et al., 2020; Painter and Qiu, 2020), political polarization (Cornelson and Miloucheva, 2020), poverty and economic dislocation (Wright et al., 2020), and differences in risk perception (Allcott et al., 2020; Barrios and Hochberg, 2020 Engle et al., 2020). These factors also influence physical distancing in the absence of government mandates (Brzezinski et al., 2020a). Our central contribution is to highlight the role of beliefs about science and attitudes regarding topics of scientific consensus in shaping patterns of physical distancing.

We leverage data on beliefs about the anthropogenic (human) causes of global warming (Howe et al., 2015) to study how physical distancing patterns vary with attitudes towards science. We combine these county-level measures with cellphone data on the movements of tens of millions of mobile devices as well as data on state-level lockdown policies. Our findings suggest that belief in science is a highly salient feature associated with varying levels of local compliance with government shelter-in-place policies. Adapting science communication to account for the target audience's bias (Jamieson and Hardy, 2014) and countering the dissemination of disinformation through modern media (Iyengar and Massey, 2019) can mitigate the risk that the message is rejected. Taken together, our results underline the importance of tailoring public health and messaging campaigns to account for local attitudes towards science.

Data We analyze differential changes in county-level movement patterns and physical distancing after the implementation of shelter-in-place policies in the United States. Daily panel data aggregated to the county-level from GPS pings of more than 40 million mobile devices, obtained from SafeGraph, allow us to track the percentage of devices that stayed home all day. The latter is defined as the ratio of the number of devices that remained home all day in a given county over the total number of devices observed. A device's home is determined as the common night-time over a 6 -week period. The underlying data was collected from various secondary sources by SafeGraph and subjected to an exhaustive 6-step process designed to guarantee reliability, granularity, anonymity and accuracy. The panel of devices in the sample is designed to be geographically and demographically representative, with a $97 \%$ correlation between the panel's population density and the American Census's population density at the county level.

To mitigate the spread of COVID-19, county and state governments implemented lockdown policies during the second half of March 2020. We collect implementation dates of state- and 
county-level school and business closures, and shelter-in-place policies, from various sources. When a policy goes into effect after $12 \mathrm{pm}$ on a given day, we assign it an implementation date one day later. We measure beliefs about science using data assembled by Howe et al. (2015) at the county-level. We focus specifically on local attitudes about the anthropogenic (human) causes of climate change. Despite scientific consensus that humans are the primary cause of present and projected climate change dynamics, there is substantial variability in local beliefs about this topic in the United States. We leverage this variation to study how patterns of physical distancing differ across counties with stronger beliefs in science (lower levels of scientific skepticism).

Theory Our main hypothesis is that counties with a higher proportion of climate change skeptics will practice less physical distancing in response to shelter-in-place policies. The mechanism for this result lies in differences in trust in scientific information and, relatedly, different risk perceptions. Those who distrust science are less likely to take estimates of the COVID threat seriously and thus will respond less to government actions targeted at increasing physical distancing. Moreover, we expect this effect to be visible over and above any divisions along party lines, despite the fact that belief in science is strongly correlated with partisan divisions in the United States (Iyengar and Massey, 2019).

Our paper enters the broader discussion about the role of information and trust in media and institutions in determining physical distancing responses. The existing literature has found that individual media consumption (Holm et al., 2020) and the rejection of conspiracy theories (Swami and Barron, 2020) are correlated with decreases in movement. Levels of trust in information from different sources vary along political lines (Bolsen and Druckman, 2018; Leiserowitz et al., 2020), and Democrats have been shown to be more inclined to believe in findings based on scientific consensus (Blank and Shaw, 2015; Hamilton et al., 2015). Our research design enables us to separate these effects and study physical distancing within partisan groups with varying beliefs in science.

Research Design We adopt a county-day level event study design to analyze physical distancing behavior depending on whether a county has a high or low proportion of people who believe in science. In line with related studies, we focus on the impact of the introduction of shelter-in-place policies on physical distancing (Brzezinski et al., 2020a; Painter and Qiu, 2020, Wright et al., 2020). The roll out of shelter-in-place mandates was staggered with states restricting physical movement at different times. The event study design allows us to investigate whether population movement was already shifting in counties prior to shelter-in-place policies and if these changes were larger in communities where belief in science was more widespread. Our research design includes county and day fixed effects to eliminate county-specific, time-invariant factors (e.g., population density, local wealth) as well as nation-wide common shocks (e.g., federal mandates, presidential announcements). We also include days-since-first-local-case fixed effects and control 
for state-wide cumulative cases and deaths, so as to eliminate the confounding effect the virus's spread might have on the relationship between policy response and physical distancing.

Our proxy for belief in science is the estimated percentage of people who state that global warming is caused by humans. Prior work establishes that belief in science differs across partisan groups in the United States. ${ }^{1}$ To account for this, we compare counties that have voted for the same party in the 2016 presidential election with each other. This implies two subsequent sample splits: first into Republican and Democratic counties, and second into counties above and below the group-specific median percentage of people who believe in global warming. We classify a county as Republican when Republican votes make up more than half of the two-party vote share. This leaves us with 491 Democratic and 2625 Republican counties. Since the medians are group specific, it is important to note that the estimates based on the sub-samples are not merely additive components of the results from the full sample. The use of group-specific medians guarantees balanced subsamples, as the distributions of belief in anthropogenic climate change for Republican and Democratic counties have limited common support (Figure SI-2).

Results We analyze how physical distancing changes with the imposition of a state-wide shelter-in-place policy. First, we compare all counties with high and low percentages of their population who believe in anthropogenic global warming. Then, we separately compare these groups for Democratic and Republican counties, to ensure that the result is not driven by party differences in perception of global warming.

Overall, we document a substantial uptake in physical distancing in most counties throughout the sample period (panel (b) in Figure SI-1). The panels on the left (a,c,e) in Figure 1 show the underlying data on the percentage of devices at home, with a polynomial trend fitted for the part of the sample above (dark) and below (light) the median belief in man-made global warming. They depict a clear difference between climate change believers and deniers in the evolution of physical distancing as the virus spread throughout March and April. This difference seems more pronounced for Democrats (c) than for Republicans (e).

The panels on the right $(b, d, f)$ show the event-study estimates with a benchmark period of 15-11 days before the policy. ${ }^{2}$ Practically all pre-trends are indistinguishable from zero at the $5 \%$ significance level, supporting the econometric validity of the research design. ${ }^{3}$ After the introduction of the policy, the share of devices that stay home completely increases by up to 5 p.p. for above-median counties than for below-median ones. Compared to an average of

\footnotetext{
${ }^{1}$ For descriptive evidence, see Figure $\overline{\text { SI-1 }}$ (panels (d) and (f)) and Figure SI-2

${ }^{2}$ Our results are very similar when using 2 days before the policy as benchmark period, as shown in Figure SI-1. We use 2 days before, as, due to the way we define the date of implementation, some lockdowns go into effect in the afternoon of day -1 . Note that this is probably the reason why there is 1 pre-trend in Figure 1 d) on day -1 that is significantly different from 0 .

${ }^{3}$ Note that our approach yields causal estimates if, conditional on our controls, counties that have not (yet) seen state-wide introductions of shelter-in-place policies are viable counterfactuals for those that did. The fact that we do not estimate significant effects before the introduction of the policy indicates that this assumption is plausible. However, even if interpreted as a descriptive correlation, our results suggest that policymakers should take into account the fact that differences in compliance are significantly associated with belief in science.
} 
approximately $25 \%$ of devices fully home in February, this implies an additional $20 \%$ increase for counties with a high share of climate change believers. When splitting the sample along party lines and comparing the effects for the counties above and below the party-specific medians, we find the differential compliance to be more pronounced for Democratic than for Republican counties. This reflects the fact that the climate change variable has both a higher minimum and a wider range across Democratic counties. Importantly, Democratic and Republican counties where scientific skepticism is high have statistically similar patterns of physical distancing after the introduction of shelter-in-place. Thus, partisan differences in the response to lockdowns appear more strongly for counties where belief in science is more pronounced.

Conclusion Beliefs about the human causes of climate change significantly influence physical distancing patterns after a local shelter-in-place policy is enacted. Importantly, these effects are present in Democratic and Republican leaning counties. These patterns highlight the importance of science education in shaping the resilience of populations to pandemics. Adapting science communication during the COVID-19 pandemic to account for the target audience's bias (Jamieson and Hardy, 2014) and countering the dissemination of disinformation through modern media (Iyengar and Massey, 2019) can help mitigate the risk that the message is rejected. 


\section{References}

Allcott, Hunt, Levi Boxell, Jacob Conway, Matthew Gentzkow, Michael Thaler, and David Y Yang (2020). "Polarization and public health: partisan differences in social distancing during the coronavirus pandemic". NBER Working Paper (w26946).

Anderson, Roy M, Hans Heesterbeek, Don Klinkenberg, and T Déirdre Hollingsworth (2020). "How will country-based mitigation measures influence the course of the covid-19 epidemic?" The Lancet 395 (10228), 931-934.

Bai, Yan, Lingsheng Yao, Tao Wei, Fei Tian, Dong-Yan Jin, Lijuan Chen, and Meiyun Wang (2020). "Presumed Asymptomatic Carrier Transmission of COVID-19". JAMA Research Letter, Published Online February 21, 2020.

Barrios, John Manuel and Yael V Hochberg (2020). "Risk perception through the lens of politics in the time of the covid-19 pandemic". University of Chicago, Becker Friedman Institute for Economics Working Paper (2020-32).

Barrios, Thomas, Rebecca Diamond, Guido W. Imbens, and Michal Kolesár (2012). "Clustering, spatial correlations, and randomization inference". Journal of the American Statistical Association $10^{\text {ry }}$ (498), 578-591. eprint: https : // doi .org/10 .1080/01621459.2012. 682524

Blank, Joshua M and Daron Shaw (2015). "Does partisanship shape attitudes toward science and public policy? the case for ideology and religion". The ANNALS of the American Academy of Political and Social Science 658 (1), 18-35.

Bolsen, Toby and James N Druckman (2018). "Do partisanship and politicization undermine the impact of a scientific consensus message about climate change?" Group Processes 86 Intergroup Relations 21 (3), 389-402.

Briscese, Guglielmo, Nicola Lacetera, Mario Macis, and Mirco Tonin (2020). "Compliance with covid-19 social-distancing measures in italy: the role of expectations and duration". National Bureau of Economic Research (preprint), Posted April 1.

Brzezinski, Adam, Guido Deiana, Valentin Kecht, and David Van Dijcke (2020a). "The covid-19 pandemic: government vs. community action across the united states". Covid Economics: Vetted and Real-Time Papers 7, 115-156.

Brzezinski, Adam, Valentin Kecht, David Van Dijcke, and Austin L Wright (2020b). "Hierarchical clustering in staggered difference-in-differences designs: a randomization inference approach".

Chen, Simiao, Juntao Yang, Weizhong Yang, Chen Wang, and Till Bärnighausen (2020). "Covid19 control in china during mass population movements at new year". The Lancet 395 (10226), 764-766.

Cornelson, Kirsten and Boriana Miloucheva (2020). "Political polarization, social fragmentation, and cooperation during a pandemic".

Engle, Sam, John Stromme, and Anson Zhou (2020). "Staying at home: mobility effects of covid-19". Covid Economics: Vetted and Real-Time Papers 4, 86-102. 
Gadarian, Shana, Sara W. Goodman, and Thomas B. Pepinsky (2020). "Partisanship, health behavior, and policy attitudes in the early stages of the covid-19 pandemic". SSRN (preprint), Posted March 2\%.

Goodman-Bacon, Andrew (2018). "Difference-in-differences with variation in treatment timing". NBER Working Paper (25018).

Grossman, Guy, Soojong Kim, Jonah Rexer, and Harsha Thirumurthy (2020). "Political partisanship influences behavioral responses to governorsâ recommendations for covid-19 prevention in the united states".

Hamilton, Lawrence C, Joel Hartter, and Kei Saito (2015). "Trust in scientists on climate change and vaccines". Sage Open 5 (3), 2158244015602752.

Holm, Eric van, Jake Monaghan, Dan C Shahar, JP Messina, and Chris Surprenant (2020). "The impact of political ideology on concern and behavior during covid-19".

Howe, Peter, Matto Mildenberger, Jennifer Marlon, and Anthony Leiserowitz (2015). "Geographic variation in opinions on climate change at state and local scales in the usa". Nature Climate Change 5, 596-603.

Hsiang, Solomon et al. (2020). "The effect of large-scale anti-contagion policies on the coronavirus (covid-19) pandemic". MedRxiv (preprint), Posted March 312020.

Hsiang, Solomon M and Amir S Jina (2014). "The causal effect of environmental catastrophe on long-run economic growth: evidence from 6,700 cyclones". Working Paper 20352. National Bureau of Economic Research.

Iyengar, Shanto and Douglas S Massey (2019). "Scientific communication in a post-truth society". Proceedings of the National Academy of Sciences 116 (16), 7656-7661.

Jamieson, Kathleen Hall and Bruce W Hardy (2014). "Leveraging scientific credibility about arctic sea ice trends in a polarized political environment". Proceedings of the National Academy of Sciences 111 (Supplement 4), 13598-13605.

Leiserowitz, Anthony, Edward Maibach, Seth Rosenthal, John Kotcher, Parrish Bergquist, Abel Gustafson, Matthew T Ballew, and Matthew Goldberg (2020). "Politics and global warming: november 2019".

Lewnard, Joseph A and Nathan C Lo (2020). "Scientific and ethical basis for social-distancing interventions against covid-19". The Lancet. Infectious diseases.

Painter, Marcus O. and Tian Qiu (2020). "Political belief affect compliance with covid-19 social distancing orders". Covid Economics: Vetted and Real-Time Papers 4, 103-123.

Swami, Viren and David Barron (2020). "Analytic thinking, rejection of coronavirus (covid-19) conspiracy theories, and compliance with mandated social-distancing: direct and indirect relationships in a nationally representative sample of adults in the united kingdom".

Viner, Russell M, Simon J Russell, Helen Croker, Jessica Packer, Joseph Ward, Claire Stansfield, Oliver Mytton, Chris Bonell, and Robert Booy (2020). "School closure and management practices during coronavirus outbreaks including covid-19: a rapid systematic review". The Lancet Child \& Adolescent Health. 
Wright, Austin L, Konstantin Sonin, Jesse Driscoll, and Jarnickae Wilson (2020). "Poverty and economic dislocation reduce compliance with covid-19 shelter-in-place protocols". University of Chicago, Becker Friedman Institute for Economics Working Paper (2020-40). 
Figure 1: Event Study Shows Compliance With Lockdown Depends on Belief in Science

(a) Data: Full Sample

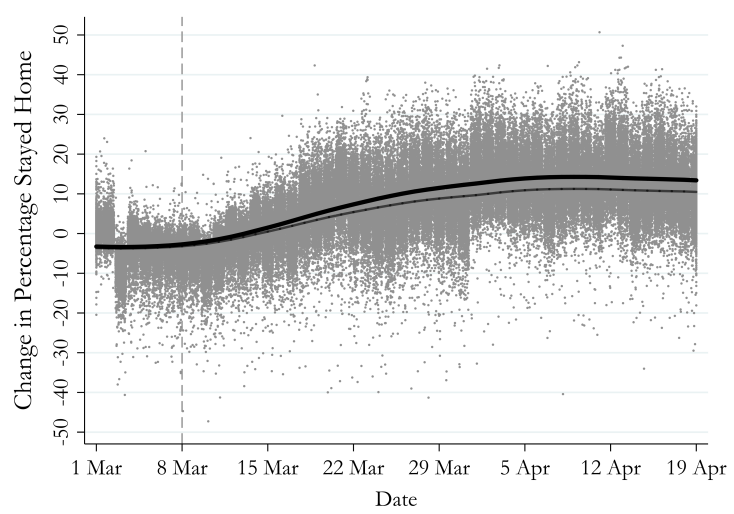

(c) Data: Democrats

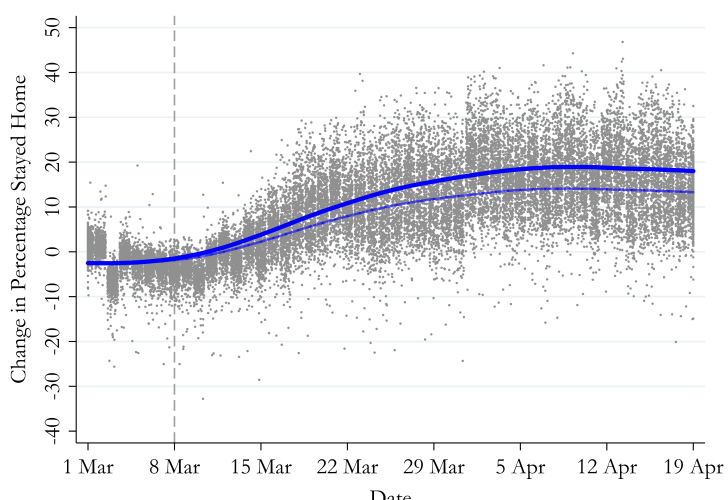

(e) Data: Republicans

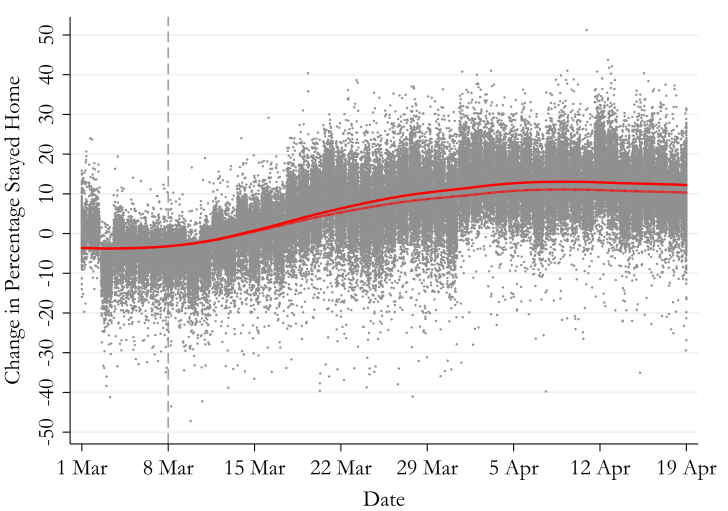

(b) Event Study: Full Sample

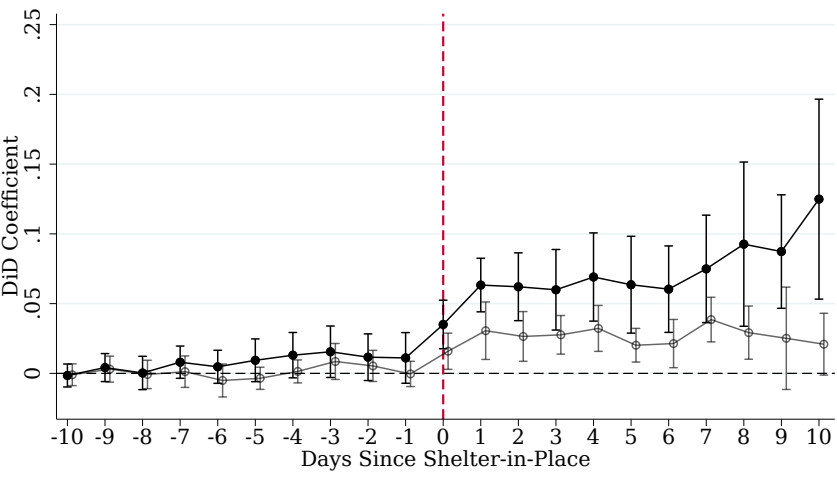

(d) Event Study: Democrats

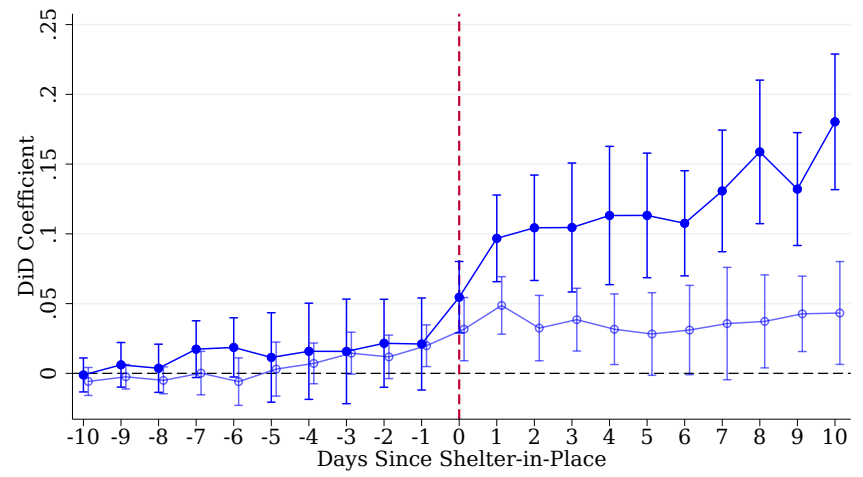

(f) Event Study: Republicans

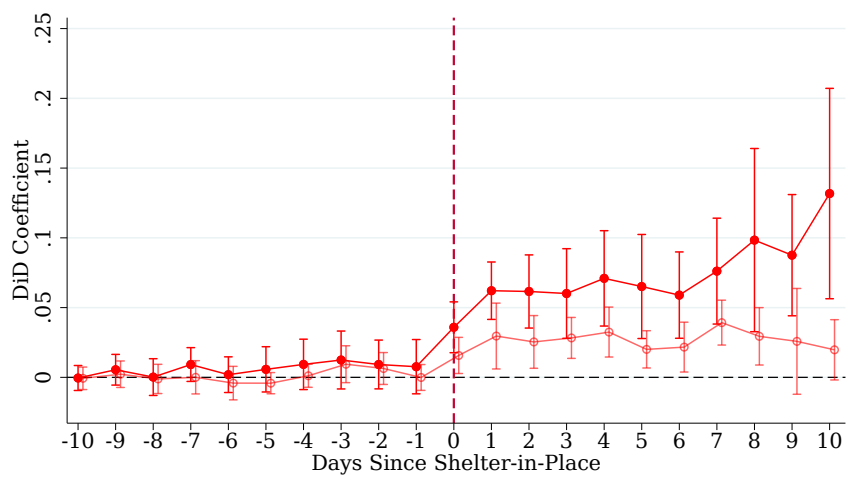

Panel a), c), e): Light: below (party-specific) median belief in man-made global warming; Dark: above-median belief in the same. Plots show the percentage difference in devices that stayed home during the sample period (March 1 to April 19) from weekdayspecific average during February. Solid lines are from local polynomial smoothing with bandwidth 5. Panel b), d), f): Light $\circ$ : below (party-specific) median belief in man-made global warming; Dark $\bullet$ : above-median believe in the same. Dependent variable: percent of devices fully at home. Difference-in-difference estimates with pre-policy event study set-up. Sample goes from 15 days before to 10 days after the policy; effect sizes shown are changes relative to the period 15-11 days before the policy. Split samples are for counties where votes for Republican (Democrat) in 2016 presidential election exceeded those for Democrat (Republican). Regressions include county-, date- and days-since-first-local-case fixed effects. Controls include state business and school closures and state-level cumulative COVID cases and deaths. $95 \%$ confidence intervals shown, based on standard errors triple-clustered by county, date and state-week. 


\section{Supporting Information}

\section{Materials and Methods}

\section{Data}

Physical Distancing. We leverage data from SafeGraph, linking GPS pings from up to 40 million mobile devices across the US. This data has previously been used in Allcott et al. (2020), Brzezinski et al. (2020a) and Painter and Qiu (2020) to study behavioral responses to the outbreak of COVID-19. ${ }^{4}$ We rely on SafeGraph's Social Distancing Metrics dataset to assess the differential responses to the outbreak of the virus and the lockdown policies at the county level. The dataset is based on an underlying panel of mobile devices in nearly all 200,000+ Census Block Groups (CBG) in the United States, which we aggregate to the county-day level. ${ }^{5}$ A device's home is defined as its common nighttime location narrowed down to Geohash-7 $(153 \mathrm{~m} \times 153 \mathrm{~m})$ precision. As our main outcome variable, we determine the percentage of devices that stayed home all day by taking the ratio of all such devices for each day over the total number observed in a county during a given month. As discussed, geographic bias of the data is small. The absolute difference between the panel's density and the true population density according to the US census remains below $1 \%$ for all counties in the sample; the correlation between both measures is 0.97 . The data is similarly representative at the county level in terms of race, demographic and income groups. ${ }^{6}$

Belief in Climate Change. To proxy belief in science, we leverage data on county-level attitudes towards climate change as described in Howe et al. (2015). In particular, we use the item human, the estimated percentage of people who think that global warming is caused mostly by human activities (see panel (f) in Figure SI-1). The data builds on 12 nationally representative climate change opinion surveys conducted between 2008 and 2013 that have been combined to an overall dataset $(n=12,061)$. Based on geographic and demographic covariates, Howe et al. (2015) apply multilevel regression and post-stratification to predict county-level opinion data. Their estimates from cross-validation and external validation exercises yield county-level error margins of \pm 8 percentage points at the $95 \%$ confidence level.

POLICIES. We compiled a comprehensive dataset on county-specific and state-wide policies from the National Governors' Association $(\mathrm{NGA})^{7}$; the National Association of Counties (NACO ${ }^{8}$;

\footnotetext{
${ }^{4}$ SafeGraph has made its data products freely available to academic researchers, see https://safegraph.com

${ }^{5}$ Only CBGs with less than 5 devices are excluded to preserve privacy.

${ }^{6}$ For a detailed exposition of SafeGraph's panel bias, see here

7 https://www.nga.org/coronavirus/\#states

8 https://ce.naco.org/?dset=COVID-19\&ind=State $\% 20$ Declaration $\% 20$ Types
} 
and Education Week, ${ }^{9}$ an independent news organization that compiled school closure data from government websites, staff reporting and the National Center for Education Statistics. This data incorporates information about the onset of school closures, closures of non-essential businesses and the introduction of shelter-in-place policies.

COVID-19 CASES AND DEATHS. County-day and state-day level information on COVID-related deaths and cases are collected by the Johns Hopkins Coronavirus Research Center. ${ }^{10}$

ELECTION RESUlts. Data on county-level vote shares by party in the 2016 presidential election was drawn from the MIT Election Lab (see panel (d) in Figure SI-1). ${ }^{11}$ We classify a county as Democratic if the Democratic vote share exceeds the Republican one (and vice versa).

County Shapefile. The maps shown in Figure SI-1 are based on the 2016 TIGER/Line county-level shapefile provided by the US Census Bureau (Department of Commerce). ${ }^{12}$

Data Availability. All data used in this manuscript are publicly available (see links on this page), except the SafeGraph data, which is proprietary but made freely available to academic researchers through the SafeGraph COVID-19 Data Consortium. ${ }^{13}$ The code for our computations is available in the supplementary materials.

\footnotetext{
${ }^{9}$ https://www.edweek.org/ew/section/multimedia/map-coronavirus-and-school-closures.html? override $=$ web

10 https://coronavirus.jhu.edu/map.html

11 https://electionlab.mit.edu/

12 https://bit.1y/2JU4ZQe.

13 https://www. safegraph. com/covid-19-data-consortium?is=5e95f848ad1cf 55efa4b30e3
} 


\section{Empirical Approach}

The following presents the event study methodology employed for the analysis of policy-related physical distancing. To disentangle differential movement patterns according to our covariates of interest, we first estimate Equation SI-1 separately for the counties with above- and belowmedian values of the variable human. However, the high correlation of climate change opinions and political leaning implies that differential effects in human may solely pick up differences in partisanship. We mitigate this concern by splitting the sample in Republican and Democratic counties. Within these groups, we further divide the sample according to the group-specific median belief in climate change and separately re-estimate Equation SI-1.

The different roll-out dates of policies across counties and states allow to construct control groups composed of areas that have not yet experienced the event. To account for the staggered treatment, we incorporate county- and day fixed effects in our analysis. On one side, this eliminates latent, time-invariant heterogeneity across counties that may be correlated with movement patterns, such as differences in income, political partisanship or geographic features. On the other side, the day fixed effects rule out the possibility that changes in physical distancing may be driven by nation-wide common shocks in terms of information or protocols.

Our specification aims at isolating the differential impact of shelter-in-place policies on physical distancing. We use an event study methodology to compare counties within states with shelter-in-place policies to those without any measure implemented. In particular, our empirical strategy follows a difference-in-differences approach with variation in treatment timing as proposed in Goodman-Bacon (2018) and previously applied to the study of behavioral responses to COVID-19 in Brzezinski et al. (2020a), Painter and Qiu (2020), and Wright et al. (2020). We obtain our main results on differential effects of the policy by estimating the following equation:

$$
p d_{c, t}=\gamma+\alpha_{c}+\delta_{t}+\sum_{j=-10}^{10} \rho_{j} D_{s, t_{0}+j}+\Psi_{\mathbf{x}_{\mathbf{c}, \mathbf{t}}}+\mathbf{\Phi}_{\mathbf{x}_{\mathbf{s}, \mathbf{t}}}+u_{c, t},
$$

where $p d_{c, t}$ is the percentage of devices that stay home all day in county $c$ at time $t . \alpha_{c}$ and $\delta_{t}$ refer to county and date fixed effects, respectively. The dummies $D_{s, t_{0}+j}$ are centered around the state policy's implementation date $t_{0}$, such that $D_{s, t_{0}+j}$ equals 1 at time $t$ if the state policy was enacted $j$ days ago. As such, we construct the dummies $D_{s, t+j}$ for the 10 days preceding as well as the 10 days following the implementation date. Panels (b,d,f) in Figure 1 plot our coefficients of interest, the sequence $\left\{\rho_{j}\right\}_{j=-10}^{10}$, for different subgroups. As a baseline period, we use the 15 to 11 th day before our state-specific policy was enacted. $\mathbf{x}_{\mathbf{c}, \mathbf{t}}$ are $K$ days-since-first-case fixed effects that are equal to 1 in period $t$ if it is $k$ days removed from the first reported COVID case in the county, and 0 otherwise, where $k \geqslant 0$. Moreover, we include the vector of covariates $\mathbf{x}_{\mathbf{s}, \mathbf{t}}$ to account for state-wide school closures, business closures, and cumulative cases and deaths. We drop those counties from the sample that moved ahead of their 
state in implementing shelter-in-place policies, so as to estimate the impact of treatment on the untreated only. Standard errors are three-way clustered by the county, day and state-week.

Randomization Inference Tests. To assess the appropriate technique to account for heteroskedasticity in our research design, we conduct several large-scale randomization inference tests (Brzezinski et al., 2020b). We extend insights from Barrios et al. (2012) to benchmark the appropriate level of clustering in our standard errors. We implement a staggered differencein-differences variation of the technique in Hsiang and Jina (2014). This approach enables us to randomly reassign actual dates of policy adoption in one (some) states to other states. We then compare the estimated effects of a benchmark specification against estimates from the randomly shuffled date-of-onset policy variable. These results suggest the two way clustering of county and day as well as three way clustering of county, day, and state-week yield highly consistent inferences about statistical precision. State-level clustering yields $t$ statistics that are significantly smaller relative the randomization test. 


\section{Supplemental Figures and Results}

Figure SI-1: Compliance With Lockdown: Robustness and Descriptive Evidence

(a) Robustness: Overall

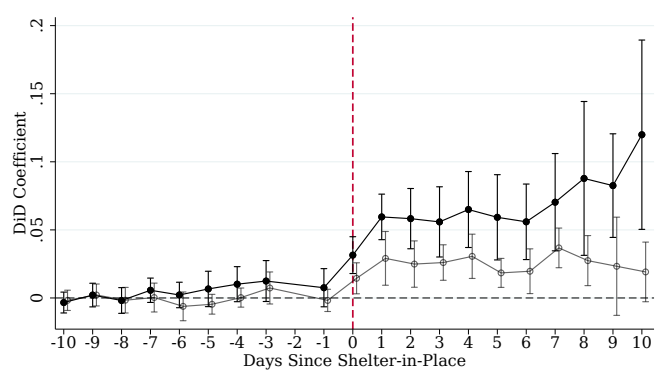

(c) Robustness: Democrats

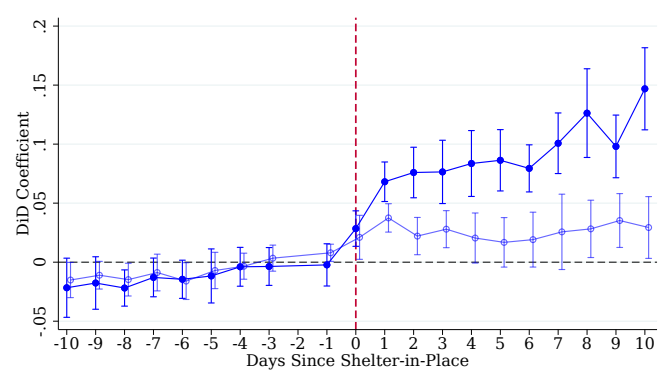

(e) Robustness: Republicans

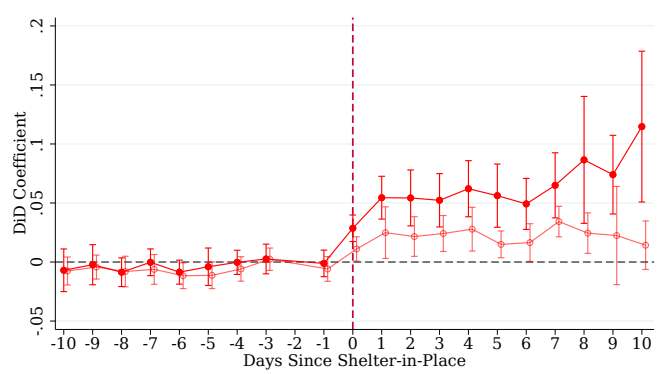

(b) Overall Change in People at Home

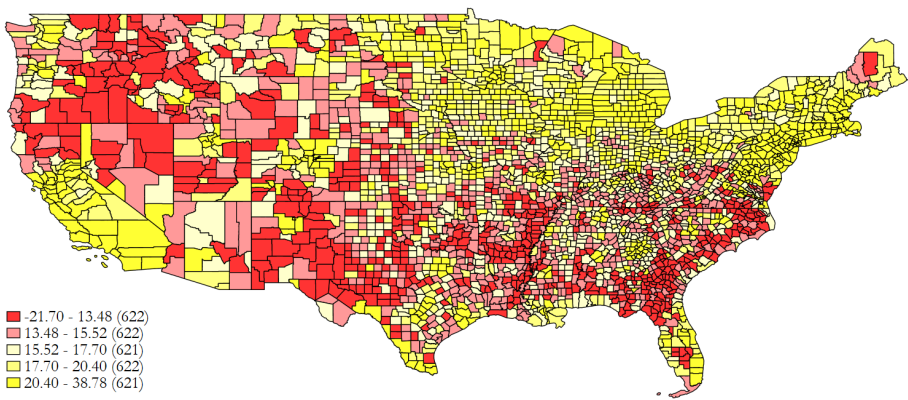

(d) Democratic Vote Share

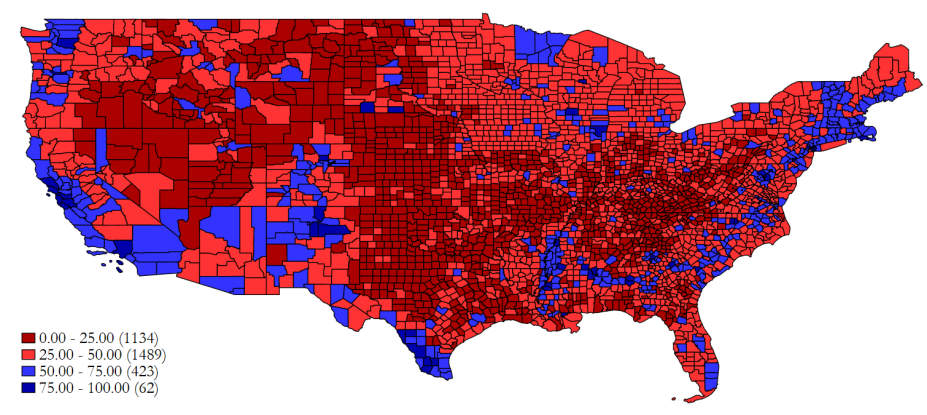

(f) Belief In Man-Made Global Warming

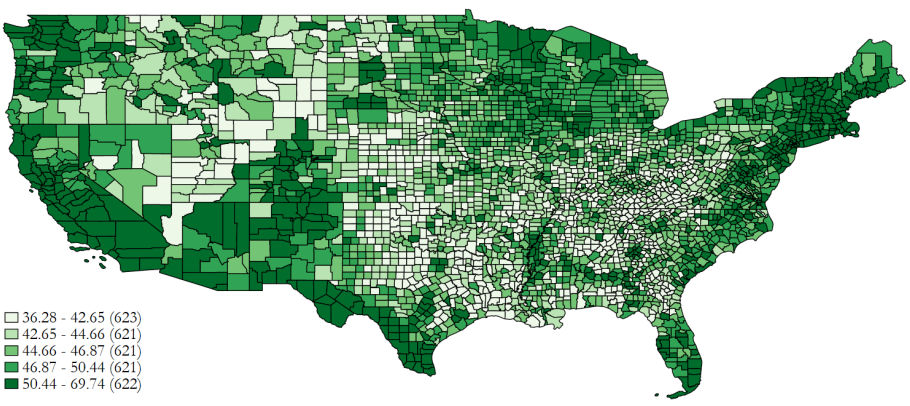

Panel a), c), e): Light $\circ:$ below (party-specific) median belief in man-made global warming; Dark $\bullet$ : above-median believe in man-made global warming. Dependent variable: percent of devices fully at home. Difference-in-differences estimates with pre-policy event study set-up. Sample goes from 10 days before to 10 days after the policy; effect sizes shown are changes relative to the period 2 days before the policy (as some lockdowns go into effect in the afternoon 1 day before). Split samples are for counties where votes for Republican (Democrat) in 2016 presidential election exceeded those for Democrat (Republican). Regressions include county-, date- and days-since-first-case fixed effects. Controls include state business and school closures and state-level cumulative COVID cases and deaths. 95\% confidence intervals shown, based on standard errors triple-clustered by county, date and state-week. Panel b): change in average percentage of devices fully at home from first week of March to first week of April. Panel d): counties classified as Republican (red) and Democratic (blue) according to their two-party vote share. Panel f): percent of people in county who believe that global warming is man-made. 
Figure SI-2: Distribution of Belief in Man-Made Global Warming, by Party

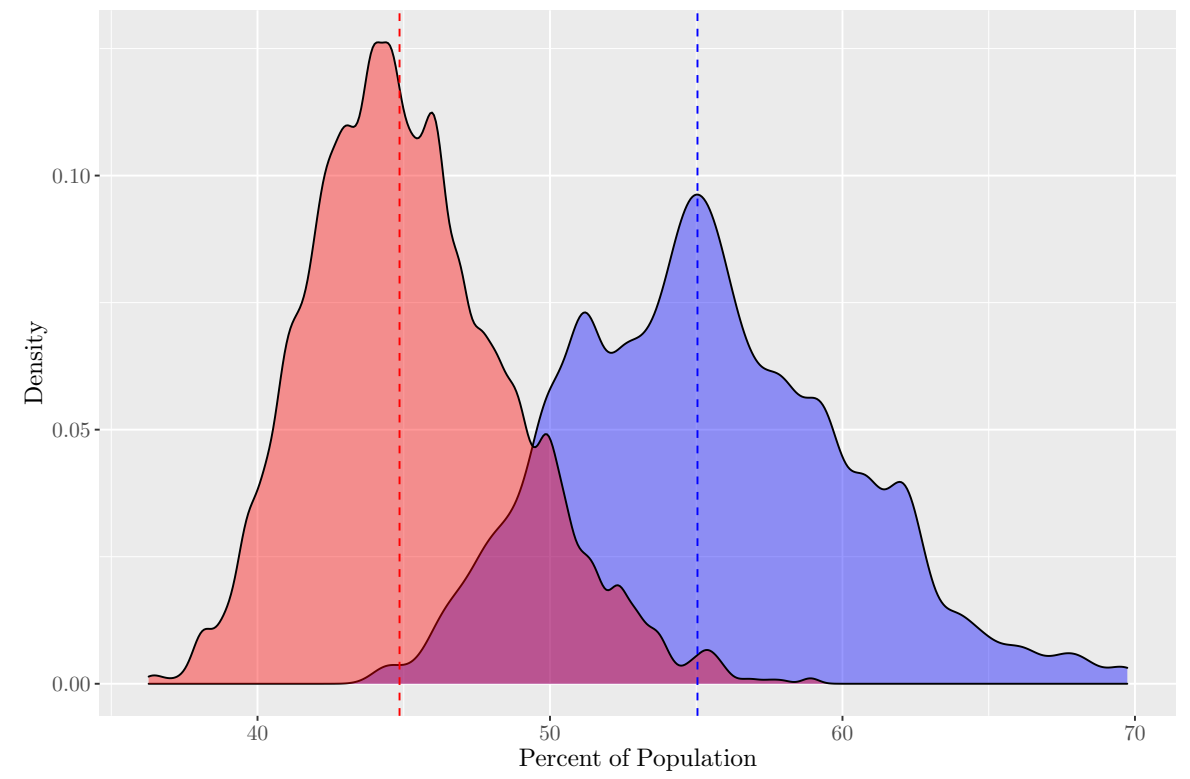

Figure shows smoothed Gaussian kernel densities using Silverman bandwith. Data comes from Howe et al. (2015) and measures percent of county population that believes in anthropogenic global warming from surveys conducted over 2008-2013. The left (red) distribution is for all Republican counties, the right (blue) distribution is for all Democratic counties. Dotted vertical lines are party-specific medians. A county is classified according to which party had a higher vote share than the other party in the 2016 presidential election. 